\title{
Anisakiasis Involving the Oral Mucosa
}

\author{
Sang Kyu Choi, \\ Cheol Keun Kim, \\ Soon Heum Kim, \\ Dong In Jo \\ Department of Plastic and Reconstructive \\ Surgery, Chungju Hospital, Konkuk \\ University School of Medicine, Chungju, \\ Korea
}

\begin{abstract}
Anisakis is a parasite with life cycles involving fish and marine mammals. Human infection, anisakiasis, occurs with the ingestion of raw infected seafood and usually presents with acute or chronic gastrointestinal symptoms from esophageal or gastric invasion. We report a rare caseinvolving the oral cavity. A 39-year-old male presented with oral and sub-sternal pain of one day duration after eating raw cuttlefish. Physical examination revealed areas of erythema and edema with a central white foreign particle on the labial and buccal mucosa. With microscopic field we could remove the foreign material from the lesions. The foreign material was confirmed to be Anisakis. Anisakis was also removed from the esophagus by esophagogastroduodenoscopy. The patient was discharged the following day without complication. Anisakiasis is frequently reported in Korea and Japan, countries where raw seafood ingestion is popular. The symptoms of acute anisakiasis include pain, nausea, and vomiting and usually begin 2-12 hours after ingestion. The differential diagnosis includes food poisoning, acute gastritis, and acute pancreatitis. A history of raw seafood ingestion is important to the diagnosis of anisakiasis. Treatment is complete removal of the Anisakis to relieve acute symptoms and prevent chronic granulomatous inflammation.
\end{abstract}

Keywords: Anisakiasis / Squid

\section{INTRODUCTION}

Anisakis is a genus of parasite with alife cycle involving fish and marine mammals. Anisakis is also known as whale worm, herring worm, and seal worm [1]. Anisakiasis is a syndromic disease characterized by acute or chronic symptoms thatoccurs when the patient ingests raw or uncooked infected seafood. Anisakiasis is typically classified into gastric, intestinal, and ectopic anisakiasis, though $95 \%$ of cases are gastric in nature [2]. Ectopic or extragastrointestinal anisakiasis is very rare and includes cases involving the oral mucosa, pleural cavity, subcutaneous tissue, and parametrium. Herein, we report a case of anisakiasis involving the oral cavity.

\footnotetext{
Correspondence: Dong In Jo

Department of Plastic and Reconstructive Surgery, Konkuk University School of Medicine, 82 Gukwon-daero, Chungju 27376, Korea

E-mail: cozarmd@kku.ac.kr

Received Sep 3, 2017 / Revised Oct 17, 2017 / Accepted Oct 18, 2017
}

\section{CASE REPORT}

A 39-year-old male presented to our clinic complaining of oral and sub-sternal pain for one day. Oral mucosal symptoms began 30 minute after the ingestion of raw cuttlefish, and sub-sternal pain began 8 hours after ingestion. Before consulting at our clinic, white thread-like foreign material had beenremoved from his oral mucosa at a local hospital. However, the patient's pain persisted, prompting his presentation at our clinic. Physical examination revealed prominent erythematous and edematous lesions on the labial and buccal mucosa. Each lesion was noted to have a centrally-placed small white foreign particle penetrating the mucosa (Figs. 1, 2). The patient's vital signs were stable. Laboratory examinations (complete blood count, white cell differential count, chemistry, electrolyte, and C-reactive protein) were unremarkable. Based on the patient's history and physical examination, we suspected anisakiasis. We consulted the department of internal medicine for evaluation of the patient's sub-sternal pain. With the 
aid of microscopic field, we were able to remove the foreign material from the lesions with a micro-forceps. The foreign material was threadlike, with a length of 3-8 $\mathrm{mm}$ and a width of $<1 \mathrm{~mm}$. In all, 8 foreign bodies were removed and were confirmed to be Anisakis larvae. Consultants from the internal medicine department performed esophagogastroduodenoscopy (EGD) and removed Anisakis larvae from the center portion of the esophagus (Fig. 3). The patient was discharged the following day without complication.

\section{DISCUSSION}

Anisakiasis is a zoonotic parasitic infection that occurs as a result of

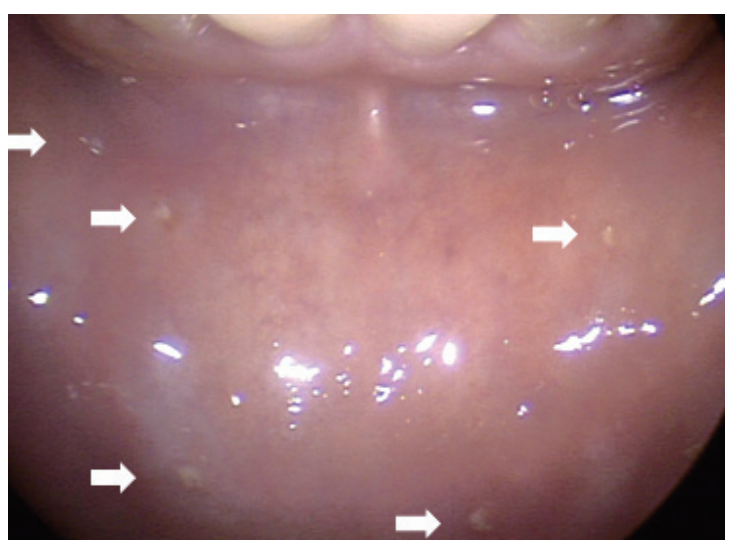

Fig. 1. Gross photograph showing mucosal invasion by the Anisakis larva (white arrows) seen as a white fragment in the lower labial mucosa.

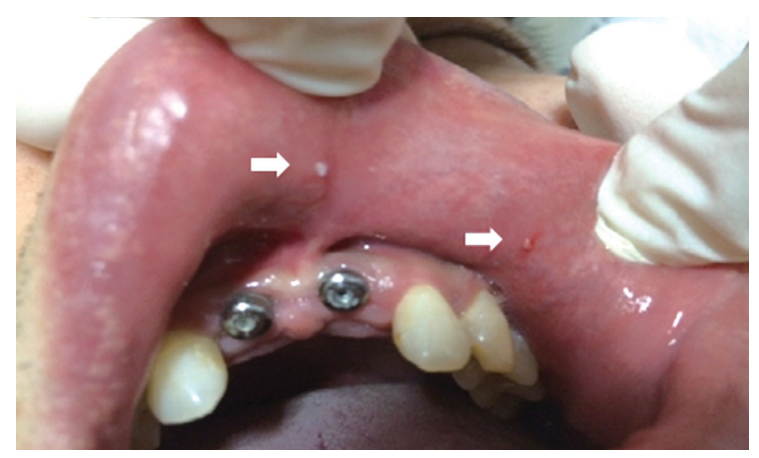

Fig. 2. Gross photograph showing mucosal invasion by the Anisakis larva (white arrows) seen as a white fragment in the upper labial mucosa.

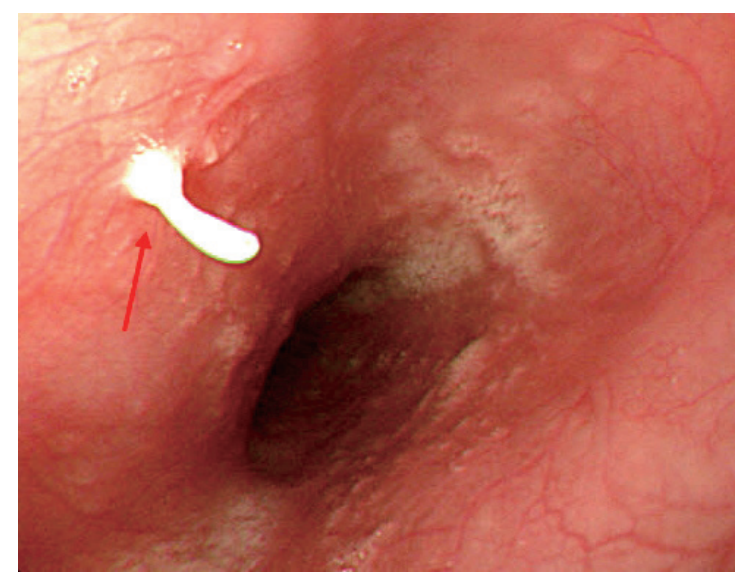

Fig. 3. Gastroscopic finding showing a white Anisakis (red arrow) invading the esophageal mucosa.

the ingestion of raw or uncooked seafood such as a sea eel, banded rudderfish, and cuttlefish. It occurs most frequently in Northeast Asia (Korea, China, and Japan) because of the popularity of raw seafood ingestion [3]. However, a recent increase in the ingestion of raw seafood in Western countries has led to an increase in the number of cases of anisakiasis reported in these areas [2].

Anisakiasis begins with the accidental human ingestion of thethird stage larvae of marine mammal parasitic nematodes belonging to the family Anisakidae. Family members Anisakis simplex and Pseudoterranova decipiens have been associated with human infection [2]. Anisakis simplex is the most common causative parasite in reports from Northwest Asia and is most commonly associated with gastric anisakiasis. On the other hand, Pseudoterranova decipiens is more common in European countries and is more commonly associated with intestinal anisakiasis. Grossly, Anisakis simplex is smaller, thinner, and more brightly colored than Pseudoterranova decipiens [2]. Our patient was infected with Anisakis simplex. Usually, when Anisakis has been ingested, the third stage larvae invade the esophageal and gastric mucosa. Acute symptoms including nausea, vomiting, epigastric pain, and low grade fever typically begin 2-12 hours after the ingestion of the infected seafood. In our case, larvae invaded the patient's oral mucosa immediately after ingestion of the infected cuttlefish, based on the onset of severe oral pain within $30 \mathrm{~min}$ utes. Chronic symptoms of anisakiasi sare dyspepsia, diarrhea, 
and epigastric pain lasting several weeks or months. Anaphylactoid reactions to larval secretions have been rarely reported [2]. Because of the vague nature of the symptoms, the differential diagnosis also includes food poisoning, acute gastritis, acute gastric ulcer, cancer, and other parasite infections. That is, severe pain occurred when sperm bags of a squid were stuck to the oral cavity after the ingestion of raw squid [4]. An eosinophilic leukocytosis is commonly found with laboratory testing; however, our patient's laboratory results were normal. We suspect that this was because the infection was in a very acute phase. In patients with chronic anisakiasis such as intestinal anisakiasis, anti-anisakis IgG/IgA antibody titers are available [2]. But, first and foremost, obtaining a careful history regarding the ingestion of raw seafood is pivotal for establishing a diagnosis of anisakiasis [1-3].

Gastroscopic or surgical removal of the larvae provides definitive diagnosis and treatment at the same time. For direct visualization of the larvae in the gastric or esophagus mucosa, EGD should be used. Generally, the invading larvae will degenerate without developing into adult worms; however, this can lead to granuloma formation [5]. Therefore, complete removal of the larvae is the treatment of choice in patients with an acute infection. The most direct method for removal is extraction of the larvae. If the larvae cannot be extracted because they are too deeply embedded in the mucosa, resection including the larvae and the mucosa is an alternative treatment.

Regarding prevention, public education about the risks of raw seafood ingestion is most important. The Food and Drug Administration recommends in United States that raw foods be blast frozen to $\leq-35^{\circ} \mathrm{C}$ for 15 hours, be normally frozen to $\leq-20^{\circ} \mathrm{C}$ for 7 days, or be heated to $\geq 70^{\circ} \mathrm{C}$, since larvae often survive at temperatures as high as $50^{\circ} \mathrm{C}$ [6]. Early evisceration of caught seafood can be recommended for preventing to migrate from the digestive tract of the captured organism to the muscle for several days. However, early evisceration may not be adequate to prevent all cases of anisakiasis because evisceration may not be performed completely. Also, in some fish infection of muscle is more prominent [7]. Discouraging the ingestion of raw seafood can also decrease the incidence of anisakiasis

Oral anisakiasis is a rare presentation of the parasitic infection anisakiasis. Severe oral pain can occur with one hour after the ingestion of raw seafood. With the aid of microscopic field, this disease can be detected and treated by a plastic surgeon.

\section{CONFLICT OF INTEREST}

No potential conflict of interest relevant to this article was reported.

\section{PATIENT CONSENT}

The patient provided written informed consent for the publication and the use of their images.

\section{REFERENCES}

1. Hochberg NS, Hamer DH. Anisakidosis: perils of the deep. Clin Infect Dis 2010;51:806-12.

2. Shimamura Y, Muwanwella N, Chandran S, Kandel G, Marcon N. Common symptoms from an uncommon infection: gastrointestinal anisakiasis. Can J Gastroenterol Hepatol 2016;2016:5176502.

3. Im KI, Shin HJ, Kim BH, Moon SI. Gastric anisakiasis cases in Chejudo, Korea. Korean J Parasitol 1995;33:179-86.

4. Shiraki Y, Kawai K, Kojima S, Suzuki Y, Ono K. Stinging in the oral cavity caused by ingestion of the sperm bags of a squid: a case report. Pathol Int 2011;61:749-51

5. Nakashima H, Akagi M, Miyabe S, Iwasawa H. Two unusual cases of a foreign body in the oral cavity caused by eating raw squid. Acta Otolaryngol Suppl 1996;522:104-7.

6. Ahmed M, Ayoob F, Kesavan M, Gumaste V, Khalil A. Gastrointestinal anisakidosis: watch what you eat. Cureus 2016;8:e860.

7. Song SB, Lee SR, Chung HH, Han NS. Infection status of anisakid larvae in anchovies purchased from local fishery market near southern and eastern sea in Korea. Korean J Parasitol 1995;33:95-9. 